\title{
ON NONRIGIDITY OF HARMONIC MAPS INTO SPHERES
}

\author{
GABOR TOTH
}

\begin{abstract}
This note studies nonrigidity of equivariant harmonic maps $f: M \rightarrow S^{n}$ of a Riemannian homogeneous space $M$ into the Euclidean $n$-sphere $S^{n}$ via representation theory applied to the induced module structure on $\mathbf{R}^{n}$ and, for specific $M$, produces (divergence-free) Jacobi fields along $f$ which do not come from isometric deformations of $f$ on the range.
\end{abstract}

1. Introduction. This note, originating from the works of R. T. Smith [5] and Do Carmo and Wallach [3], continues the studies [7-9] on rigidity of a harmonic map $f: M \rightarrow S^{n}, n \geqslant 2$, with constant energy density $e(f)=\lambda / 2, \lambda \in \operatorname{Spec}(M)$, of a compact Riemannian manifold $M$ into the Euclidean $n$-sphere $S^{n}$ via the (finitedimensional) vector space $K(f)$ of all divergence-free Jacobi fields along $f$. Recall [4] that $f$ is harmonic iff $\Delta^{M} f=\lambda \cdot f$. Furthermore, by translating tangent vectors of $S^{n} \subset \mathbf{R}^{n+1}$ to the origin of $\mathbf{R}^{n+1}$, a vector field $v$ along $f$ gives rise to a vector function $\hat{v}: M \rightarrow \mathbf{R}^{n+1},\langle\hat{v}, f\rangle=0$. Then [9], $v \in K(f)$ iff $\Delta^{M} \hat{v}=\lambda \cdot \hat{v}$.

The vector space $\operatorname{so}(n+1) \circ f$ of infinitesimal isometric deformations of $f$ is a linear subspace of $K(f)$ and $f$ is said to be rigid if $\operatorname{so}(n+1) \circ f=K(f)$. The importance of $K(f)$ is shown by the generalized Do Carmo-Wallach classification theorem [9] which states that, for oriented (isotropy) irreducible Riemannian homogeneous $M$, the equivalence classes of full harmonic maps $f: M \rightarrow S^{n}$ with $e(f)=$ $\lambda / 2$ can be parametrized by a compact convex body lying in $K\left(f_{\lambda}\right) / \operatorname{so}\left(V_{\lambda}\right) \circ f_{\lambda}$, where $f_{\lambda}: M \rightarrow S^{n(\lambda)}$ (= unit sphere in $V_{\lambda}$ ) is a standard minimal immersion given by an orthonormal base in the eigenspace $V_{\lambda}$ corresponding to $\lambda$. Though there is no immediate generalization of this classification to aribitrary Riemannian homogeneous $M$ the object of this note is to show that the occurrence of rigidity is rare. In $\S 2$, we reformulate the condition of rigidity in terms of representation theory. This is then applied in $\S 3$ to prove nonrigidity of harmonic maps in various instances.

\section{Properties of rigid harmonic maps.}

THEOREM 1. Let $M=G / K$ be a compact naturally reductive Riemannian homogeneous space (with base point $o=\{K\}$ ) and $f: M \rightarrow S^{n}, n \geqslant 2$, a full rigid harmonic map with $e(f)=\lambda / 2, \lambda \in \operatorname{Spec}(M)$. Then (a) $f$ is equivariant with respect to an orthogonal G-module structure on $\mathbf{R}^{n+1}$; (b) $\mathbf{R}^{n+1}$ is an irreducible G-submodule of $V_{\lambda}$;

Received by the editors August 3, 1984.

1980 Mathematics Subject Classification. Primary 58E20; Secondary 58D15, 22E46. 
(c) $\operatorname{dim} \operatorname{Fix}\left(K, \mathbf{R}^{n+1}\right)=1,2$ or 4 and $\mathbf{R}^{n+1}$ is a real, complex or quaternionic G-module, accordingly; (d) $S^{2}\left(\mathbf{R}^{n+1}\right)\left(=\right.$ symmetric square of $\left.\mathbf{R}^{n+1}\right)$ is the sum of irreducible $G$-submodules which are class 1 for $(G, K)$.

Proof. (a) See [9].

(b) Let $\mathbf{R}^{n+1}=V \oplus W$ be a nontrivial orthogonal $G$-invariant decomposition. Then $f=(g, h)$, where $g: M \rightarrow V$ and $h: M \rightarrow W$ are equivariant, in particular, $\|g\|^{2}$ and $\|h\|^{2}\left(=1-\|g\|^{2}\right)$ are constants. As $f$ is full we have $\|g\|=\cos t$ and $\|h\|=\sin t$ for some $0<t<\pi / 2$. The vector function $\hat{v}=(-\tan t \cdot g, \cot t \cdot h)$ : $M \rightarrow \mathbf{R}^{n+1}$ gives rise to an element $v \in K(f)$ which, by fullness of $f$, does not belong to so $(n+1) \circ f$. Thus, $\mathbf{R}^{n+1}$ is irreducible and mapping the $i$ th base vector to the $i$ th component of $f$ induces a $G$-module monomorphism $\mathbf{R}^{n+1} \rightarrow V_{\lambda}$.

(c) Setting $F=\operatorname{Fix}\left(K, \mathbf{R}^{n+1}\right)$, we first note that the orthogonal complement $f(o)^{\perp}$ of $f(o)$ in $F$ corresponds bijectively to the set of $G$-invariant vector fields along $f$. Assuming that $\operatorname{dim} F \geqslant 2$, let $\hat{v}_{o} \in f(o)^{\perp},\left\|\hat{v}_{o}\right\|=1$, and denote by $v$ the corresponding $G$-invariant vector field along $f$. Then $[8] v \in K(f)$ and so $\hat{v}=J \cdot f$, where $J \in \operatorname{so}(n+1)$. We claim that $J$ is a complex structure on $\mathbf{R}^{n+1}$, i.e. $J^{2}=-I_{n+1}$ $\left(I_{n+1}=\right.$ identity of $\left.\mathbf{R}^{n+1}\right)$. Indeed, setting $\hat{u}=\left(J^{2}+I_{n+1}\right) \cdot f$, we have $\langle\hat{u}, f\rangle=0$ and so $\hat{u}$ induces a vector field $u$ along $f$ which automatically belongs to $K(f)=$ so $(n+1) \circ f$. Thus, by fullness of $f, J^{2}+I_{n+1}$ is skew and hence zero. By $G$-invariance of $v, J$ commutes with the action of $G$ on $\mathbf{R}^{n+1}$. For $\operatorname{dim} F \geqslant 3$ we proceed analogously by selecting an orthonormal base $\left\{\hat{v}_{o}^{i}\right\}_{i=1}^{p} \subset f(o)^{\perp}, \operatorname{dim} F=p+1$, and considering the corresponding anticommuting family $\left\{J^{i}\right\}_{i=1}^{p}$ of linearly independent skew-symmetric complex structures on $\mathbf{R}^{n+1}$. By simple argument (involving $J^{1} \cdot J^{2}$ ), it follows that $p \geqslant 3$. On the other hand, endowing $\mathbf{R}^{n+1}$ with the complex structure $J^{1}$ and applying Schur's lemma to $J^{2} J^{3}$, we have $J^{2} J^{3}=\alpha I_{n+1}+\beta J^{1}, \alpha$, $\beta \in \mathbf{R}$. By simple algebra, $\alpha=0$ and $\beta= \pm 1$. The rest is clear.

(d) The $G$-submodule $W_{f}=\operatorname{span}\left\{f(x)^{2} \mid x \in M\right\} \subset S^{2}\left(\mathbf{R}^{n+1}\right)$ splits into the sum of class 1 subrepresentations for $(G, K)$. We claim that $W_{f}=S^{2}\left(\mathbf{R}^{n+1}\right)$. Indeed, setting $B \in W_{f}^{\perp} \subset S^{2}\left(\mathbf{R}^{n+1}\right)$ the vector function $\hat{w}=B \cdot f$ satisfies $\langle\hat{w}, f\rangle=0$ and hence gives rise to an element $w$ of $K(f)$. By rigidity, $B$ is skew and hence zero.

REMARKS. (1) If $K$ acts on $T_{o}(M)$ (via the isotropy representation) without nonzero fixed vectors (e.g. if $M$ is irreducible) then $\operatorname{dim} F=\operatorname{dim} \operatorname{Fix}\left(K, \mathbf{R}^{n+1}\right)=1$. Indeed, assume that $\hat{v}_{o} \in f(o)^{\perp},\left\|\hat{v}_{o}\right\|=1$, exists and consider the corresponding $G$-invariant vector field $v$ and complex structure $J$ on $\mathbf{R}^{n+1}$. Choose a Killing vector field $X$ on $M$ such that $f_{*}(X)=Y \circ f$ for a nonzero $Y \in \operatorname{so}(n+1)$. Setting $\hat{u}=J \cdot Y \cdot f$, as $v$ is orthogonal to $\operatorname{im}(f) \subset S^{n}$, we have $\langle\hat{u}, f\rangle=-\langle Y \cdot f, J \cdot f\rangle=$ $-\left\langle f_{*}(X)^{\hat{n}}, \hat{v}\right\rangle=0$. Hence $\hat{u}$ induces a vector field $u \in K(f)$ along $f$ and, by rigidity, $J \cdot Y=Z \in \operatorname{so}(n+1)$. Taking transposes, it follows that $J$ and $Y$ anticommute. On the other hand, $J$, being a $G$-module automorphism, commutes with $Y$, i.e. $J \cdot Y=0$. This implies $Y=0$, a contradiction.

(2) As Professor Joseph A. Wolf has informed the author, for $M=G / K$ compact irreducible symmetric with rank $M=\operatorname{rank} G$, the symmetric square of every irreducible component of $V_{\lambda}, \lambda \in \operatorname{Spec}(M)$, is the sum of class 1 subrepresentations for $(G, K)$. 
A partial converse of Theorem 1 is given as follows:

TheOREM 2. Let $M=G / K$ be a compact irreducible Riemannian homogeneous space and $\lambda \in \operatorname{Spec}(M)$ such that $V_{\lambda}$ is irreducible, the $K$-module $V_{\lambda} \mid K$ has multiplicity 1 decomposition into irreducible components and $S^{2}\left(V_{\lambda}\right)$ is the sum of class 1 subrepresentations for $(G, K)$. Then the standard minimal immersion $f_{\lambda}: M \rightarrow S^{n(\lambda)}$ is rigid.

Proof. Given $v \in K\left(f_{\lambda}\right)$ we have $\hat{v}=B \cdot f_{\lambda}$ for some matrix $B$ since the components of $f_{\lambda}$ form a basis in $V_{\lambda}$. We may assume that $B$ is symmetric (by splitting $B$ into symmetric and skew-symmetric parts if necessary). As $0=\left\langle\hat{v}, f_{\lambda}\right\rangle=$ $\left\langle B \cdot f_{\lambda}, f_{\lambda}\right\rangle$, we have $B \in W_{f_{\lambda}}^{\perp}$, where $W_{f_{\lambda}}=\operatorname{span}\left\{f_{\lambda}(x)^{2} \mid x \in M\right\} \subset S^{2}\left(V_{\lambda}\right)$. On the other hand, as $V_{\lambda} \mid K$ has multiplicity 1 decomposition, we can apply the argument of Do Carmo and Wallach in the proof of 4.2. Lemma in [3, pp. 50-51], and it follows that $W_{f_{\lambda}}$ is the sum of all class 1 subrepresentations of $(G, K)$ in $S^{2}\left(V_{\lambda}\right)$. Then, by hypothesis, $W_{f_{\lambda}}=S^{2}\left(V_{\lambda}\right)$ which implies that $B=0$.

RemarK. For $M$ rank 1 symmetric and $\lambda \in \operatorname{Spec}(M)$ the first two assumptions on $V_{\lambda}$ are automatically satisfied (cf. [1,6]) and so $f_{\lambda}$ is rigid iff $S^{2}\left(V_{\lambda}\right)$ is the sum of class 1 subrepresentations for $(G, K)$. In particular, for

$$
(G, K)=(\mathrm{SO}(m+1), \mathrm{SO}(m)),
$$

$f_{\lambda}$ is rigid iff $m=2$ (Calabi's rigidity [2]) or $f_{\lambda}$ is of degree 1 (isometry [9]). For $(G, K)=(S U(m+1), S(U(m) \times U(1))), \quad m \geqslant 2$, and $(G, K)=(\operatorname{Sp}(3), \operatorname{Sp}(2) \times$ $\mathrm{Sp}(1)$ ), any standard minimal immersion $f_{\lambda}$ of degree $\geqslant 4$ is nonrigid by the results of Urakawa in [10].

\section{Applications.}

THEOREM 3. Let $f: M_{1} \times M_{2} \rightarrow S^{n}$ be a full harmonic map with constant energy density of the Riemannian product of compact naturally reductive Riemannian homogeneous spaces $M_{1}$ and $M_{2}$. If $f$ does not factor through the canonical projections $\pi_{i}$ : $M_{1} \times M_{2} \rightarrow M_{i}, i=1,2$, then $f$ is nonrigid.

Proof. Assuming that $f$ is rigid, by Theorem $1, f$ is equivariant with respect to an irreducible $G_{1} \times G_{2}$-module structure on $\mathbf{R}^{n+1}$, where the compact Lie group $G_{i}$ acts transitively on $M_{i}$ by isometries. Then $\mathbf{R}^{n+1}=V_{1} \otimes V_{2}$, where $V_{i}$ is an irreducible $G_{i}$-module over $\mathbf{R}, \mathbf{C}$, or $\mathbf{H}$ according as $\operatorname{dim} \operatorname{Fix}\left(K, \mathbf{R}^{n+1}\right)=1,2$ or 4 . In particular, $f(o)=v_{1} \otimes v_{2}, v_{i} \in V_{i}$, and, by equivariance, $f\left(x_{1}, x_{2}\right)=f_{1}\left(x_{1}\right) \otimes f_{2}\left(x_{2}\right), x_{i} \in M_{i}$, where $f_{i}: M_{i} \rightarrow V_{i}$. As $f$ does not factor through $\pi_{i}$, we have $\operatorname{dim} V_{i} \geqslant 2$ so that we can select nonzero matrices $A_{i} \in \operatorname{so}\left(V_{i}\right)$. Define the vector function $\hat{u}: M_{1} \times M_{2} \rightarrow$ $\mathbf{R}^{n+1}$ by $\hat{u}\left(x_{1}, x_{2}\right)=\left(A_{1} \otimes A_{2}\right),\left(f_{1}\left(x_{1}\right) \otimes f_{2}\left(x_{2}\right)\right), x_{i} \in M_{i}$. Then $\langle\hat{u}, f\rangle=0$ and $\hat{u}$ induces a vector field $u \in K(f)$ along $f$. By rigidity and fullness of $f, A_{1} \otimes A_{2}$ is skew, a contradiction.

EXAMPLE. As an easy computation (involving the spectrum of $S^{2} \times S^{3}$ ) shows, the canonical projection $\pi_{1}: S^{2} \times S^{3} \rightarrow S^{2}$ followed by the Veronese map $f_{\lambda_{2}}: S^{2} \rightarrow S^{4}$ is rigid. 
THEOREM 4. Given a compact naturally reductive Riemannian homogeneous space $M$ and $\lambda \in \operatorname{Spec}(M)$, the number of equivalence classes of full rigid harmonic maps $f$ : $M \rightarrow S^{n}$ with $e(f)=\lambda / 2$ does not exceed the number of inequivalent irreducible components of $V_{\lambda}$.

Proof. Given an irreducible component $\mathbf{R}^{n+1}$ in $V_{\lambda}$ we have to show that any two full rigid equivariant harmonic maps $f, f^{\prime}: M \rightarrow S^{n}$ with $e(f)=e\left(f^{\prime}\right)=\lambda / 2$ are equivalent. This is done by using the uniqueness of $K$-fixed vectors over $\mathbf{R}, \mathbf{C}$ or $\mathbf{H}$.

\section{REFERENCES}

1. M. Berger, P. Gauduchon and E. Mazet, Le spectre d'une variété Riemannienne, Lecture Notes in Math., vol. 194, Springer-Verlag, Berlin, 1971.

2. E. Calabi, Minimal immersions of surfaces in Euclidean spheres, J. Differential Geom. 1 (1967), 111-125.

3. M. Do Carmo and N. Wallach, Minimal immersions of spheres into spheres, Ann. of Math. (2) 93 (1971), 43-62.

4. J. Eells and L. Lemaire, A report on harmonic maps, Bull. London Math. Soc. 10 (1978), 1-68.

5. R. T. Smith, Harmonic mappings of spheres, Thesis, Warwick University, England, 1972.

6. The spherical representations of groups transitive on $S^{n}$, Indiana Univ. Math. J. 24 (1974), $307-325$.

7. G. Toth, On rigidity of harmonic mappings into spheres, J. London Math. Soc. (2) 26 (1982), 475-486.

8. On naturally reductive homogeneous spaces harmonically embedded into spheres, J. London Math. Soc. (2) 29 (1984), 175-180.

9. Harmonic and minimal maps with applications in geometry and physics, E. Horwood Series, Halsted Press, Wiley, New York, 1985.

10. H. Urakawa, Minimal immersions of projective spaces into spheres (to appear).

11. N. R. Wallach, Minimal immersions of symmetric spaces into spheres, Symmetric Spaces, Dekker, New York, 1972, pp. 1-40.

Department of Mathematics, Ohio State University, 231 West 18th Avenue, Columbus, Ohio 43210 